\title{
Análisis de la gestión administrativa que brinda a los usuarios el área de balcón de servicios del Banco Bank Ecuador, sucursal Santo Domingo de los Tsáchilas.
}

\section{Analysis of the administrative management that provides users with the balcony area of services of the Bank branch, Ecuador, Pichincha.}

Evelyn Karina Tinoco Diaz. ${ }^{1}$ Marisol Lisseth Guanuchi Pucha ${ }^{2}$, Katya Vanessa Macías Badaraco ${ }^{3}$, Ramiro Enrique Guamán $\mathrm{Chavez}^{4} \&$ Efraín Velastegui López

\section{DOI: https://doi.org/10.33262/visionariodigital.v3i1.131}

\section{Resumen.}

La investigación está relacionada al análisis a la gestión administrativa que brinda a los usuarios el área de balcón de servicios del Banco Bank Ecuador, sucursal Santo Domingo de los Tsáchilas, proyecto que nace al ver las necesidades y las deficiencias detectadas en los procesos del Banco Bank Ecuador, este estudio investigativo está enfocado específicamente en el área de balcón de servicios, se tomó en cuenta la opinión de los clientes ya que ellos son los indicados para calificar el servicio que brindan las instituciones bancarias y determinar si se estaba proporcionando un servicio con amabilidad, cortesía, confiabilidad, rapidez y eficiencia ya que se observa en algunos funcionarios de estas instituciones la falta de cordialidad en la atención de los clientes, esa inadecuada actitud en el desempeño de sus funciones influye en el deterioro de su imagen, la misma que representa uno de los factores primordiales en la decisión de los clientes, se pretende lograr describir el estado administrativo y operativo real del mismo y de esta manera proponer la solución acorde a sus necesidades para ello se realizó una

\footnotetext{
${ }^{1}$ Universidad Técnica Luis Vargas Torres, Facultad de Ciencias Administrativas, Santo Domingo, Ecuador, kaevecris@hotmail.com

${ }^{2}$ Universidad Técnica Luis Vargas Torres, Facultad de Ciencias Administrativas, Santo Domingo, Ecuador, liss.leo@hotmail.com

${ }^{3}$ Universidad Técnica Luis Vargas Torres, Facultad de Ciencias Administrativas, Santo Domingo, Ecuador, katybada_16@hotmail.com

${ }^{4}$ Universidad Técnica Luis Vargas Torres, Facultad de Ciencias Administrativas, Santo Domingo, Ecuador, ramiro.guaman@utelvt.edu..ec 
investigación con base a la aplicación de las técnicas como encuestas a los diversos niveles del Banco, entrevistas y observación directa para establecer factores como: conocimiento, ambiente de control, actividades de control, establecimiento de funciones y responsabilidades entre otros, que determinan la calidad de gestión administrativa que tiene el Banco y recoger criterios de aspectos relevantes tanto al interior y a lo exterior, así se identificó el problema principal como es " Deficiente gestión administrativa que se brinda a los usuarios del área de balcón de servicios del Banco Bank Ecuador, sucursal santo Domingo de los Tsachilas”.

\section{Introducción.}

Muchas de las funciones de los bancos son guardar fondos, prestar dinero, garantizar préstamos y brindar un excelente servicio al cliente que es el principal en la actualidad bancaria. Para ellos analizar la forma de obtener y captar la mayor cantidad de clientes, significa elemento clave para el funcionamiento y tomando en cuenta que Ecuador se enfrentan a una fuerte competitividad debido a que existen distintos establecimientos bancarios que prestan los mismos servicios dentro del mercado, así las instituciones crediticias se preocupan por el servicio al cliente que puedan brindar.

A sí mismo el objetivo de esta investigación, es dar a conocer a las instituciones bancarias la importancia del servicio, se facilitará por medio de un modelo que describa los servicios al cliente que se deben brindar en las agencias bancarias, identificando errores que actualmente existen en las empresa y empleados relativo al servicio al cliente.

Siendo el servicio el que ligará a la empresa con sus clientes así poder adecuar una relación interpersonal establecida entre la empresa y el cliente, se desarrolló el presente trabajo de investigación de tipo descriptivo, y para obtener datos sobre como prestan el servicio al cliente, se utilizó una encuesta dirigida a 396 de los usuarios, así como a los servidores que laboran en el área de Balcón de Servicios del Bank Ecuador Sucursal Santo Domingo de los Tsachilas con sus respectivas jefaturas.

Se podrá diagnosticar la gestión que brinda a sus usuarios el Área de Balcón de Servicios del Banco Bank Ecuador Sucursal Santo Domingo de los Tsachilas así como el desempeño y profesionalismo de cada uno de los servidores.

En los actuales momentos en Banco Bank Ecuador Sucursal Santo Domingo de los Tsachilas registra un alto índice de usuarios que solicitan los múltiples servicios que ofrece esta entidad Publica Financiera, enfocándose directamente en el área de Balcón de Servicios debemos mencionar que registra en el año 2017 y lo que va del año 2018 incremento de usuarios para hacer uso de sus productos como la apertura de cuentas de ahorro y corrientes, certificados de integración de capital, transferencias, tramites por préstamos de Desarrollo Humano, depósitos en general a título personal.

Podemos mencionar que uno de los inconvenientes para los servidores que laboran en la Sucursal Santo Domingo no presten una atención personalizad al usuario es la falta de capacitación continua en temas inherentes a sus gestiones, brindando una atención precaria y 
negativa a los clientes, debido a mantener equipos obsoletos sin tecnología de punta, así como también un área de trabajo reducida no acorde a las necesidades del servidor y del usuario.

Como consecuencia de todo esto podemos encontrarnos con un usuario insatisfecho por un servicio deficiente que lo único que genera es una imagen institucional deteriorada con múltiples quejas de usuarios por no ser atendidos con un servicio óptimo.

Las empresas se deben caracterizar por el altísimo nivel en la calidad de los servicios que entrega a los clientes que nos compran o contratan. La calidad de los servicios depende de las actitudes de todo el personal de la empresa. El servicio es la solución de un problema o la satisfacción de una necesidad. El personal en todos los niveles y áreas debe ser consciente de que el éxito de las relaciones entre la empresa y cada uno de los clientes depende de las actitudes y conductas que observen en la atención de las demandas de las personas que son o representan al cliente (Reyes, 2014).

Por lo general todo cliente evalúa la calidad del servicio que ofrecen las instituciones y más aún cuando nos referimos a instituciones financieras públicas, ya que de acuerdo al trato y servicio que se le brinde al usuario se generara el nivel de satisfacción, y las expectativas con las que se acercó a la institución serán de su complacencia.

En síntesis, toda empresa que logre la satisfacción del cliente obtendrá como beneficios: 1) La lealtad del cliente (que se traduce en futuras ventas), 2) difusión gratuita (que se traduce en nuevos clientes) y 3) una determinada participación en el mercado, 4) Crecimiento y expansión y 5) Generación de conocimiento (Hernandez, 2014).

El servicio al cliente, hoy por hoy, es identificado como una fuente de respuestas a las necesidades del mercado y las empresas; debe contemplar una estrategia más dinámica; mas ahora cuando así lo exige el contexto. Por ello, instituciones de servicio sobretodo, como las financieras y las entidades bancarias, deben emprender estos retos. (Peralta, 2010)

La falta de información interna y externa en el banco; hace que se incrementen los límites en la comunicación con el cliente interno y/o externo, motivo por el que, sin duda, se acentúa la falta de satisfacción al cliente, por lo que éste ignora muchas veces el verdadero compromiso de servicio del Banco, así como las diferentes facilidades en la atención del Banco con él, lo cual, además; no permite el flujo de comunicación del cliente habitual con el que no lo es. (Peralta, 2010). Los clientes son cada vez más exigentes, ya no sólo buscan precio y calidad, sino también, una buena atención, un ambiente agradable, comodidad, un trato personalizado, un servicio rápido. Si un cliente queda insatisfecho por el servicio o la atención, es muy probable que hable mal de uno y cuente de su mala experiencia a otros consumidores. Si un cliente recibe un buen servicio o atención, es muy probable que vuelva a adquirir nuestros productos o que vuelvan a visitarnos. Si un cliente recibe un buen servicio o atención, es muy probable que nos recomiende con otros consumidores. Es primordial tomar en cuenta dichos aspectos, ya que, si se logran entender adecuadamente cada uno de ellos y, aplicarlos de la manera correcta se logrará tener una ventaja competitiva (Sonora, 2013). 
Cuando los clientes no se sienten satisfechos con los productos que adquieren o reciben, tienen dos opciones: pueden quejarse, o pueden dejarlo estar, como si no hubiera pasado nada. Si el usuario no comunica los motivos de su queja, de su decepción o desagrado, prácticamente no deja a la empresa la oportunidad de estar al corriente de su insatisfacción. Por el contrario, los clientes que se quejan siguen hablando con nosotros, dándonos la oportunidad de devolverles a un estado de satisfacción para que sigan siendo consumidores de nuestros productos. (Ongallo, 2012)

Las reclamaciones de los clientes son una de las fuentes que pueden incluso convertirse en la base de los programas de mejora de calidad y servicio de un buen número de empresas y organizaciones de venta directa. Debemos ser capaces de recibir a estos clientes que protestan con los brazos abiertos y motivarles para que se acerquen a nosotros con sus comentarios. Para ello serán claves los denominados «momentos de la verdad», en los que la interacción con el cliente. (Ongallo, 2012)

El hecho de que los clientes estén logrando más al demandar un mejor servicio y que se hayan vuelto más sofisticados al reconocerlo, no quiere decir que todas las organizaciones profesionales hayan "saltado» al tren de la calidad. La realidad es muy diferente. Algunas organizaciones son las líderes de esa revolución, otras le siguen los pasos y algunas duermen mientras los cambios se producen. (Cottle, 2010)

La insatisfacción y/o una mala o deficiente calidad de servicio no generan necesariamente infidelidad o ruptura total con la entidad financiera, aunque sí es más probable que incrementen la ruptura parcial, apreciándose una cierta inercia a mantenerse como cliente de la entidad, muchas veces por comodidad u otros motivos. (Santiago, 2006)

A fin de lograr la aceptación por parte del cliente, una compañía debe proporcionarle exactamente lo que desea en el momento que lo requieren. En consecuencia, una firma debe hacer todo lo posible para identificar sus necesidades y satisfacerlas.

Restablecer el servicio es hacer que nuestro cliente sonría, incluso después de que cometimos un error, Es resolver el problema del cliente y que este salga del establecimiento con la impresión de que está tratando con el mejor Banco del país, quizás retribuir al cliente no signifique un gasto importante, pero la publicidad de boca en boca que este haga si podría generar un ingreso millonario . (Tschohl, 2013)

Podremos encontrar un banco en donde esperar es un placer, no solo porque cuenta con unas instalaciones, maravillosas, abiertas e iluminadas, sino por que maneja la expectativa de sus clientes, les informa con exactitud en cuanto tiempo serán atendidos. Un banco donde la gente no sale de mal genio, sino con una sonrisa, de eso se trata el servicio al cliente (Vallejo, 2017).

En la actualidad el Bank Ecuador sucursal Santo Domingo de lo Tsachilas, gracias a la estratégica ubicación que mantiene en el centro de la cuidad de Santo Domingo, denominada zona bancaria, cuenta a su alrededor con varias instituciones financieras públicas y privadas.

Desde sus inicios ha fomentado las actividades en la zona urbana rural y sus alrededores, relacionada al comercio formal, informal, sector ganadero, agropecuario, microempresas, 
artesana de consumo y desarrollo, entre otros más, aprovechando los recursos naturales y las bondades con que cuenta el subsuelo de esta provincia de Santo Domingo de los Tsachilas.

En los tiempos actuales el desarrollo acelerado de nuestra Provincia ha hecho que el Banco Bank Ecuador haya visto un incremento acelerado de clientes que buscan en la institución satisfacer sus necesidades financieras, calidad en el servicio, seguridad y confiabilidad, por tanto, la institución debería invertir en ofrecer capacitaciones motivadoras a sus servidores, mantener el equipo de servicios profesionales necesarios, tecnologías y equipos de óptima calidad.

La Sucursal Santo Domingo cuenta actualmente con 38 servidores publicos distribuidos en los diferentes departamentos, cuenta con una infraestructura de hace aproximadamente 64 años, por lo cual el área de balcón der servicios es bastante reducida y no presta ventilación adecuada para atender a los usuarios.

\section{Metodología.}

\section{Tipo de investigación.}

Se considera el análisis crítico donde se buscan las opciones más recomendables para poder realizar cambios positivos en lo referente a la gestión administrativa que brinda a sus usuarios el are de balcón de servicios del Bank Ecuador Sucursal Santo Domingo de los Tsachilas ya que en la actualidad no permite al usuario o cliente encontrar una atención especializada con calidad de servicio, como tampoco ofrece instalaciones adecuadas para que el cliente o usuario encuentre un ambiente acogedor que le permita de manera cómoda espera su turno para realizar las transacciones respectivas.

\section{Población y muestra.}

Dentro de esta investigación científica la población involucra a las personas que se encuentran inmersas dentro del presente proceso, por lo que consideramos a los usuarios/clientes que hacen uso o solicitan los productos que ofrece el Área de Balcón de Servicios y sus servidores respectivamente del área mencionada en Banco Bank Ecuador sucursal Santo Domingo de los Tsáchilas.

Con la matriz muestral se determina el tamaño de la muestra que se va a obtener a través de las siguiente formula que se detalla:

Para obtener la información requerida se procederá a utilizar las siguientes técnicas:

Encuesta: Para esta técnica realizaremos la encuesta a 396 usuarios tomados de la matriz muestra, mismos que visitan las instalaciones del Área de Balcón de Servicios del Bank Ecuador Sucursal Santo Domingo de los Tsáchilas y conocer sus expectativas en cuanto a la atención personalizada y de calidad que desean recibir, así como también un lugar cómodo y acogedor donde realicen sus transacciones, es muy importante emplear con sabiduría las encuestas. 
Entrevista: Se procederá a entrevistar a los servidores que laboran en el Área de Balcón de Servicios del Banco Bank Ecuador Sucursal Santo Domingo de los Tsachilas, con sus respectivas jefaturas para determinar la prioridad que mantienen en solicitar la contratación de más personal y la ampliación de la mencionada área de trabajo.

Instrumentos: Guías para entrevista, hoja de cuestionario, material bibliográfico, revisión de informes, entre otros.

Análisis de datos: se realizará con tabulación para analizar estadísticamente, analiza y explora los mismos, frecuencia y porcentajes.

Se han utilizado el método descriptivo, cualitativo y propositivo.

Tabla 1: Resultado de la encuesta a Clientes del Bank Ecuador.

\begin{tabular}{|c|c|c|c|c|}
\hline \multicolumn{5}{|c|}{ ENTREVISTA A JEFATURAS } \\
\hline $\mathbf{N}^{\circ}$ & ITEMS & PARAMETROS & FREC & $\%$ \\
\hline \multirow{2}{*}{\multicolumn{2}{|c|}{$\begin{array}{l}1 \text { ¿ Considera usted que cuenta el número de personal adecuado } \\
\text { para atender diariamente a los usuarios/ clientes del Área de } \\
\text { Balcón dervicios? }\end{array}$}} & SI & 2 & $100 \%$ \\
\hline & & NO & 0 & $0 \%$ \\
\hline \multirow{2}{*}{\multicolumn{2}{|c|}{$\begin{array}{l}\text { ¿Considera usted que el Área de Balcón de servicios cuenta con } \\
2 \text { el espacio adecuado y comodidad para la atención de los } \\
\text { usuarios/ clientes? }\end{array}$}} & SI & 1 & $50 \%$ \\
\hline & & NO & 1 & $50 \%$ \\
\hline \multirow{2}{*}{\multicolumn{2}{|c|}{$\begin{array}{l}\text { ¿La institución capacita continuamente a los servidores en lo } \\
3 \text { relacionado a la atención al usuario/ cliente y relaciones } \\
\text { humanas? }\end{array}$}} & SI & 2 & $100 \%$ \\
\hline & & NO & 0 & \\
\hline \multirow{4}{*}{\multicolumn{2}{|c|}{$\begin{array}{l}4 \text { ¿El sistema de tecnología y las herramientas de computación de } \\
\text { uso son óptimas para la atención de los usuarios/clientes? }\end{array}$}} & SI & 1 & $50 \%$ \\
\hline & & NO & 1 & $50 \%$ \\
\hline & & & CAl & TACION \\
\hline & & & ESPECIFIC & \\
\hline \multirow{4}{*}{\multicolumn{2}{|c|}{$\begin{array}{l}\text { ¿Qué cambios sugiere realizar en el Área de balcón de Servicios } \\
\text { para mejorar la atención de los usuarios/clientes y servidores } \\
\text { internos? }\end{array}$}} & SI & b) & AR LA \\
\hline & & SI & TECNOLO & \\
\hline & & & $\begin{array}{l}\text { c) } \mathrm{MA} \\
\mathrm{EN}\end{array}$ & $\begin{array}{l}\text { RSONAL } \\
\text { ITICAS }\end{array}$ \\
\hline & & $\mathrm{NO}$ & d) OTR & \\
\hline
\end{tabular}

Elaborado por: Grupo de investigadores.

De acuerdo a los datos proporcionados sobre la pregunta relacionada a la atención el 54,54\% de los encuestados expresaron su inconformidad con el servicio brindado.

Respecto a la pregunta sobre si las instalaciones son adecuadas para recibir el servicio el $78,28 \%$ de los usuarios encuestados dieron una respuesta positiva ya que si creen ellos que son _apropiadas para una atención de calidad. 
Cabe recalcar que los clientes a pesar de no estar conforme con los servicios una vez realizado su trámite el 54,54\% se siente conforme a pesar de que la atención no es excelente.

Con relación a la pregunta número cuatro sobre el personal que atiende a los usuarios un 89,14\% de las personas encuestadas dice que el Área de Balcón de Servicios no cuenta con el suficiente personal para atender los requerimientos de los ciudadanos, debido a esto están de

\section{Encuesta a clientes}

\begin{tabular}{|c|c|c|c|c|}
\hline $\mathbf{N}^{\mathbf{0}}$ & Ítems & Parámetros & Frec & $\%$ \\
\hline 01 & $\begin{array}{l}\text { ¿recibe usted una atención de } \\
\text { calidad en el área de balcón de } \\
\text { servicios? }\end{array}$ & $\begin{array}{l}\text { Si } \\
\text { No }\end{array}$ & $\begin{array}{l}180 \\
216\end{array}$ & $\begin{array}{l}45,46 \% \\
54,54 \%\end{array}$ \\
\hline \multirow[t]{2}{*}{02} & $\begin{array}{l}\text { ¿ cree usted que las instalaciones } \\
\text { del área de balcón de servicios } \\
\text { donde solicita los servicios es la } \\
\text { adecuada para una atención de } \\
\text { calidad? }\end{array}$ & Si & 310 & $78,28 \%$ \\
\hline & & No & 86 & $21,72 \%$ \\
\hline 03 & $\begin{array}{l}\text { ¿ se siente usted satisfecho por la } \\
\text { atención prestada en el área de } \\
\text { balcón de servicios de la sucursal } \\
\text { santo domingo? }\end{array}$ & Si & 180 & $54,54 \%$ \\
\hline 04 & $\begin{array}{l}\text { ¿ considera que el área de balcón } \\
\text { de servicios cuenta con el } \\
\text { personal necesario para atender } \\
\text { sus requerimientos }\end{array}$ & No & 353 & $10,86 \%$ \\
\hline 05 & $\begin{array}{l}\text { ¿ le gustaría contar con un sistema } \\
\text { de atención que le permita mejorar } \\
\text { su tiempo de espera? }\end{array}$ & $\mathrm{Si}$ & 370 & $93,43 \%$ \\
\hline & & No & 26 & $6,57 \%$ \\
\hline
\end{tabular}

acuerdo en un 93,43\% que si es necesario mejorar el sistema de atención del Área de Balcón de Servicios del Banco Bank Ecuador Sucursal Santo Domingo de los Tsáchilas.

Tabla 1: Resultado de Encuesta a las Jefaturas del Bank Ecuador. 


\begin{tabular}{|c|c|c|c|c|}
\hline \multicolumn{5}{|c|}{ ENCUESTA A CLIENTES } \\
\hline $\mathbf{N}^{\mathbf{o}}$ & ITEMS & $\begin{array}{l}\text { PARAME } \\
\text { TROS }\end{array}$ & FREC & $\%$ \\
\hline \multirow[t]{2}{*}{1} & ¿Recibe usted una atención de calidad en el Área de & SI & 180 & $45,46 \%$ \\
\hline & Balcón de servicios? & NO & 216 & $54,54 \%$ \\
\hline \multirow[t]{2}{*}{2} & $\begin{array}{l}\text { ¿ Cree usted que las instalaciones del Área de } \\
\text { Balcón de Servicios donde solicita los servicios es la }\end{array}$ & & 310 & $78,28 \%$ \\
\hline & adecuada para una atención de calidad? & $\begin{array}{c}\text { SI } \\
\text { NO }\end{array}$ & 86 & $21,72 \%$ \\
\hline \multirow[t]{3}{*}{3} & $\begin{array}{l}\text { ¿ Se siente usted satisfecho por la atención prestada } \\
\text { en el Área de Balcón de Servicios de la Sucursal }\end{array}$ & & 216 & $54,54 \%$ \\
\hline & Santo Domingo? & SI & & \\
\hline & & NO & 180 & $45,46 \%$ \\
\hline \multirow[t]{3}{*}{4} & $\begin{array}{l}\text { ¿ Considera que el Área de Balcón de servicios } \\
\text { cuenta con el personal necesario para atender sus }\end{array}$ & & 43 & $10,86 \%$ \\
\hline & requerimientos & SI & & \\
\hline & & NO & 353 & $89,14 \%$ \\
\hline \multirow{3}{*}{5} & ¿ Le gustaría contar con un sistema de atención que & & 370 & $93,43 \%$ \\
\hline & le permita mejorar su tiempo de espera? & SI & & \\
\hline & & $\mathrm{NO}$ & 26 & $6,57 \%$ \\
\hline
\end{tabular}

Elaborado por: Grupo de investigadores.

El 100\% de los entrevistados de las Jefaturas consideran que cuentan con el número de personal adecuado para atender diariamente a los usuarios/clientes del Área de Balcón de Servicios, podemos observar que esta se encuentra en contraposición a la pregunta $\mathrm{N}^{\mathrm{a}} 1$ de la encuesta realizada a los usuarios.

Así mismo el 50\% manifestaron que el espacio es adecuado y cómodo para que funcione el Área de Balcón de Servicio para la atención de los usuarios/clientes.

Los entrevistaron manifestaron que si se capacita constantemente los servidores en lo relacionado a la atención al usuario/cliente y relaciones humanas.

La opinión de los entrevistados se encuentra dividida en relación a la calidad de los sistemas tecnológicos y herramientas de computación usados para la atención a los usuarios/clientes.

Las sugerencias vertidas por los entrevistados para realizar en el Área de Balcón de Servicios un mejoramiento en la atención de los usuarios/clientes fueron:

- Capacitación especifica

- Mejorar la tecnología

- Más personal en Áreas criticas 
Tabla 2:Resultados de Encuesta a los servidores de Bank Ecuador.

\section{Entrevista a servidores}

\begin{tabular}{|c|c|c|c|c|}
\hline $\mathrm{N}^{\circ}$ & Items & Parametros & Frec & $\%$ \\
\hline \multirow[t]{2}{*}{01} & $\begin{array}{l}\text { ¿ se siente usted satisfecho con el } \\
\text { trabajo que realiza en el área de } \\
\text { balcón de servicios? }\end{array}$ & $\mathrm{Si}$ & 30 & $78,94 \%$ \\
\hline & & No & 8 & $21,06 \%$ \\
\hline \multirow[t]{2}{*}{02} & $\begin{array}{l}\text { ¿ la infraestructura física donde } \\
\text { usted ejerce su función, le permite } \\
\text { realizar un trabajo de calidad? }\end{array}$ & $\mathrm{Si}$ & 23 & $60,52 \%$ \\
\hline & & No & 15 & $39,48 \%$ \\
\hline \multirow[t]{2}{*}{03} & $\begin{array}{l}\text { ¿ cree usted que las autoridades del } \\
\text { bank ecuador le apoyan en sus }\end{array}$ & $\mathrm{Si}$ & 20 & $52,63 \%$ \\
\hline & $\begin{array}{l}\text { iniciativas para mejorar la gestión } \\
\text { institucional? }\end{array}$ & No & 18 & $47,37 \%$ \\
\hline 04 & $\begin{array}{l}\text { ¿se siente usted satisfecho con los } \\
\text { recursos tecnológicos con que } \\
\text { cuenta para la atención diaria al } \\
\text { usuario/ cliente? }\end{array}$ & No & 25 & $65,79 \%$ \\
\hline 05 & $\begin{array}{l}\text { ¿la institución lo ha capacitado } \\
\text { continuamente para brindar una } \\
\text { atención eficiente y de calidad al } \\
\text { usuario/cliente? }\end{array}$ & No & 17 & $44,73 \%$ \\
\hline
\end{tabular}

Elaborado por: Grupo de investigadores.

Los servidores publicos encuestados en un 78,94\% manifestaron sentirse satisfechos con el trabajo que realizan en el Área de Balcón de Servicios.

Respecto a la infraestructura, los servidores publicos manifestaron en un $60,52 \%$ si les permite realizar un trabajo de calidad.

La pregunta relacionada con el apoyo que las autoridades brindan a los servidores en sus iniciativas para mejorar la gestión institucional, el 52,63\% manifestaron sentirse apoyados, sin 
embrago hay que recalcar que un porcentaje considerable del $47,37 \%$ expresaron su inconformidad.

En consideración al personal operativo el 65,79\% manifestaron no sentirse satisfechos con los recursos tecnológicos que tiene para desempeñar su tarea y para la atención diaria al usuario/cliente.

Los encuestados expresaron el 55,27\% que a pesar de recibir capacitación del Bank Ecuador estas son de manera general y no dirigidas a su especialidad y función que desempeñan en el puesto de trabajo que está desempeñando al momento.

\section{Conclusiones.}

- Los procesos que se desarrollan actualmente en el Banco Bank Ecuador, tiene baja participación de valor agregado, sus tiempos de ejecución mantienen tiempos excesivos de respuesta, lo que hace improductivo el accionar general de los Departamentos del Bank Ecuador, reflejándose en las quejas de los usuarios.

- El análisis de la cadena de valor es una herramienta gerencial poderosa que ayudara al Bank Ecuador a identificar fuentes de ventaja competitiva. Sin embargo, este análisis no mejorar los procesos por si solo, sino que debe haber un fuerte compromiso institucional, esto es desde Gerente hasta el último de los funcionarios, caso contrario no tiene sentido iniciar una filosofía de mejorar continuo.

- La propuesta plantea disminuye los tiempos, incrementa el valor agregado, lo que hace suponer que vale la pena implementar el mejoramiento de los procesos sugeridos a través de la aplicación de estas estrategias y metodologías planteadas en este trabajo.

\section{Bibliografía.}

Cottle, D. (2010). El servicio centrado en el cliente: como lograr quie regresen y sigan utilizando sus servicios. Ediciones Diaz de Santos.

Hernandez, C. (2014). El servicio al Cliente como factor de competitividad y calidad en las empresas de servicio. Ciencia Administrativa, 11.

Ongallo, C. (2012). La atencion al cliente y el servicio postventa. Ediciones Diaz de Santos.

Peralta, W. (2010). Estrategias del Servicio al cliente. El Cid Editor.

Reyes, P. (2014). Calidad del Servicio para aumentar la satisfaccion del cliente. Mexico.

Santiago, M. ,. (2006). La calidad de servicio bancario: entre la fidelidad y la rutura. Madrid: Universidad Complutense.

Sonora, I. T. (2013). Importancia de la calidad del servicio al cliente. El buzon de Pacioli, 36.

Tschohl, J. (2013). Como conservar clientes con un buen servicio. Mexico.

Vallejo, y. S. (2017). Servicio con pasion . Colombia: Penguin Ramdom. 


\section{Para citar el artículo indexado.}

Tinoco E., Guanuchi M., Macías K., Guamán R. \& Velasteguí E. (2019). Análisis de la gestión administrativa que brinda a los usuarios el área de balcón de servicios del Banco Bank Ecuador, sucursal Santo Domingo de los Tsáchilas. Revista electrónica Visionario Digital 2(2), 16-28. Recuperado desde: http://cienciadigital.org/revistacienciadigital2/index.php/VisionarioDigital/article/view/131/1 $\underline{15}$

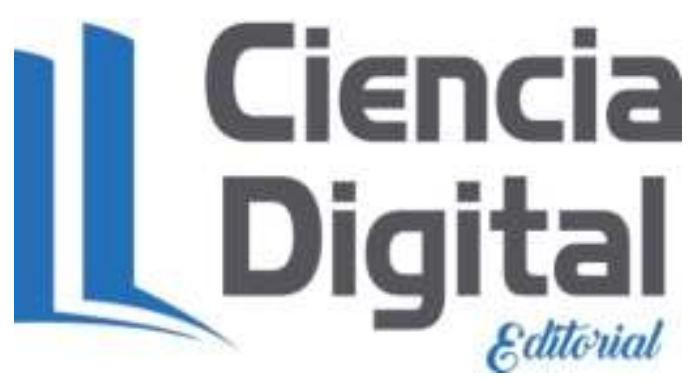

El artículo que se publica es de exclusiva responsabilidad de los autores y no necesariamente reflejan el pensamiento de la Revista Ciencia Digital.

El articulo queda en propiedad de la revista y, por tanto, su publicación parcial y/o total en otro medio tiene que ser autorizado por el director de la Revista C
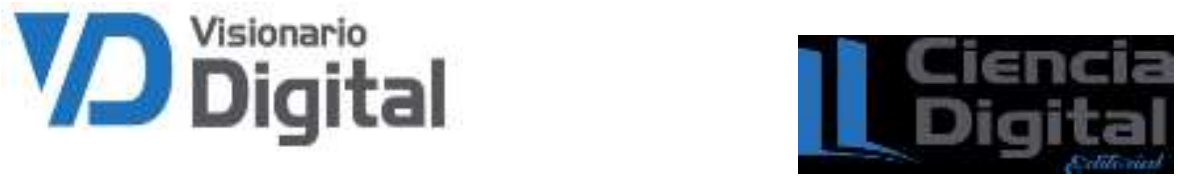